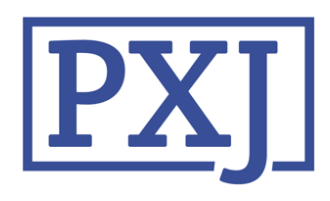

Patient Experience Journal

\title{
The impact of queue-controlled modified open access scheduling on no-show rate in a community mental health child psychiatry med check clinic: A pilot study
}

Timothy Lesaca MD

Trinity Healthcare

Follow this and additional works at: https://pxjournal.org/journal

Part of the Health Services Administration Commons

\section{Recommended Citation}

Lesaca T. The impact of queue-controlled modified open access scheduling on no-show rate in a community mental health child psychiatry med check clinic: A pilot study. Patient Experience Journal. 2017; 4(1):141-144. doi: 10.35680/2372-0247.1193.

This Case Study is brought to you for free and open access by Patient Experience Journal. It has been accepted for inclusion in Patient Experience Journal by an authorized editor of Patient Experience Journal. 


\section{The impact of queue-controlled modified open access scheduling on no-show rate in a community mental health child psychiatry med check clinic: A pilot study}

\section{Cover Page Footnote}

The author wishes to thank Mercy Behavioral Health and Pittsburgh Mercy Health System administration and staff, and in particular Joel Cockley, Anna Boettcher MD, and Brittany Kail for their invaluable assistance with data collection and article edification. 


\title{
The impact of queue-controlled modified open access scheduling on no- show rate in a community mental health child psychiatry med check clinic: A pilot study Timothy Lesaca MD, Pittsburgh Mercy Health System, tlesaca@hotmail.com
}

\begin{abstract}
No-shows in child and adolescent psychiatry are a particularly concerning phenomenon that have ramifications beyond productivity and mental health outcomes. One might contend that children have a fundamental right to health care, and the failure to address the no-show phenomenon passively impedes that human right. In this study, a queue-controlled modified open access scheduling model (QCMOAS) was compared to a traditional scheduling model for the incidence of no-shows in a community mental health child psychiatric med check clinic. A six month period of QCMOAS was compared to the preceding six months of traditional scheduling and the six month period exactly one year prior to QCMOAS. Z test was used for statistical significance. For the six month period immediately after the implementation of OCMOAS, a $7.32 \%$ decrease in no-show rate was observed, and compared to the six month period exactly 1 year prior to QCMOAS, a decrease of $6.38 \%$ was noted. In both cases the result was statistically significant. Preliminary interpretation of this data suggests that the employment of OCMOAS significantly decreased the no-show rate in this community mental health center's child and adolescent med check clinic. The findings are strengthened by the relatively large number of scheduled appointments reviewed and by the fact that statistical significance appeared to be unrelated to seasonal scheduling patterns. Limitations of this pilot study and recommendations for future investigation are discussed.
\end{abstract}

\section{Keywords}

No-shows, open access scheduling, queue-control, child psychiatry, community mental health, med check

\section{Acknowledgments}

The author wishes to thank Mercy Behavioral Health and Pittsburgh Mercy Health System administration and staff, and in particular Joel Cockley, Anna Boettcher MD, and Brittany Kail for their invaluable assistance with data collection and article edification.

\section{Introduction}

Numerous studies have concluded that there is a severe shortage of child and adolescent psychiatrists, which in combination with unacceptably high no-show rates in community mental health centers, worsens an already serious problem in providing mental health services to populations of lower socioeconomic status ${ }^{1,2}$. Psychiatric no-shows occur with a reported frequency as high as $60 \%$ and are associated with disruption of treatment, exacerbation of symptoms, functional impairment, and increased risk for psychiatric hospitalization ${ }^{3.4}$. From this perspective, finding a way to lower the no-show rate should be considered a child welfare concern of the greatest importance.

Studies of no-show patterns have found a direct association between the waiting time for an appointment and the likelihood of not attending ${ }^{5}$. One particularly important study conducted in a large urban community mental health center found that reducing the wait time for psychiatric appointments from 13 days to 0 days resulted in the no-show rate decreasing from $52 \%$ to $18 \%{ }^{6}$.

As waiting times for psychiatric appointments have increased and the shortage of child psychiatrists has worsened, there has been increased interest in applying open access scheduling models to psychiatric appointment scheduling ${ }^{7}$. In contrast to the traditional scheduling model in which psychiatric appointments are booked weeks or months in advance, open access appointment scheduling is a method in which patients are offered an appointment on the same day that they call or at the time of their choosing, preferably within 24-48 hours. In theory, this results in minimal wait time for appointments, increased scheduling flexibility, and decreased no-show ${ }^{8}$.

The open access model applies the principle of queuing in an effort to match the demand for appointments with the availability of the clinician. Demand and capacity must be properly balanced, however, for open access scheduling to be successful. Given that patient demand is highly difficult

Patient Experience Journal, Volume 4, Issue 1 - Spring 2017

(C) The Author(s), 2017. Published in association with The Beryl Institute and Patient Experience Institute

Downloaded from www.pxjournal.org 
to predict, open access scheduling has the potential to fail if not somehow customized to accommodate the unpredictability of client requests for appointments ${ }^{9}$. Both traditional and open access models for psychiatric med check scheduling are built around the tenet that appointments are scheduled before the client runs out of medication. In traditional scheduling, the patient queue is predictable since appointments are scheduled well in advance, but the model is inefficient, whereas in open access scheduling there is greater efficiency, but the queue is unpredictable and difficult to control.

A practical method to predict the patient queue would be to monitor each patient's supply of medication. By creating a time window of a few days prior to running out of medication in which patients would be allowed or reminded to reschedule, an open access scheduling system could be implemented that controlled against unpredictable patient demand. The risk of losing patients to follow-up would also be greatly decreased since a medication supply monitoring system would create its own queue of patients that a clinic could reach out to in the event that an appointment had not been scheduled.

In this pilot study, what will be termed 'queue-controlled modified open access scheduling' (QCMOAS), which utilizes a client med refill/scheduling tracker, will be compared to a traditional scheduling model for the incidence of child and adolescent psychiatric med check no-shows.

\section{Methods}

This pilot study was conducted at Mercy Behavioral Health, an urban community mental health center in Pittsburgh, Pennsylvania. The variable for investigation was the rate of psychiatric med check no-shows in one child and adolescent psychiatrist's outpatient clinic. Initial psychiatric evaluations and psychotherapy visits were not included in this study. The time intervals for comparison were the 12 months prior to the initiation of QCMOAS scheduling, divided into 6 month increments, and the 6 month period immediately after the initiation of QCMOAS. The age range for children within the clinic was 4 years old to 18 years old. Per clinic protocol, all identified patients were required to be accompanied by a parent or legal guardian, unless otherwise legally emancipated.

February 1, 2016 was designated as the transition date at which time the clinic would change from its traditional scheduling model to QCMOAS. Prior to the transition date, psychiatric med checks were scheduled from 4 to 16 weeks in advance, with an appointment reminder by telephone call one business day prior to the scheduled appointment. Medication refills without client attendance were strongly discouraged, but exceptions were made on a case-to-case basis.

During the three transition months leading up to the implementation of QCMOAS, a centralized appointment telephone number was established and a scheduling staff was recruited and trained in preparation for the new scheduling protocol. During this transition, no appointments were scheduled beyond February 1, 2016. Rather than scheduling in advance at the most recent psychiatric med check, clients were requested to call the central appointment number 7 to 10 days prior to running out of medication, at which point they would be provided an appointment within 3-5 business days. Clients were informed that as of the transition date, medications could no longer be provided without having a face to face meeting with either the doctor or nurse, and case-to-case exceptions would no longer be allowed. Clients were informed of the transition date, and were provided an appointment card with the central appointment number and the date on which they are being requested to call. Clients were also informed that urgent or emergency med checks could be scheduled using the same central appointment number. In anticipation of the potential that the psychiatrist's schedule might become filled, a morning walk in clinic staffed by a CRNP for one hour each morning Monday to Friday was created in the scheduling template.

QCMOAS scheduling began formally as planned. On the day clients called, a psychiatric med check appointment was offered within 3-5 days with the client's assigned child and adolescent psychiatrist or sooner if the client was dealing with an emergency. If there was no availability in the psychiatrist's schedule within the time frame needed, or if the client anticipated running out of medication before an appointment with the psychiatrist could be accommodated, then an appointment in the same day walk in clinic was offered. If these options were found to not be acceptable, the client was asked to call the next work day, at which point the psychiatrist might have availability for a same day appointment, or the client could then choose to utilize the walk in clinic.

As part of OCMOAS, a client med refill/scheduling tracker report was run each work day for the psychiatrist's patient caseload. This information was obtained from an integrated software program that combined the electronic prescribing history and the clinic scheduling template. The tracker report showed the number of days remaining on the patient's prescription and if the patient already had an appointment date scheduled.

For clients with a preference for text messaging, a text reminder to reschedule was sent to those unscheduled patients identified as having 7 days of medication remaining, and was repeated 3 work days later for those 
who had yet to reschedule. For clients without text message availability or preference, a single telephone call rescheduling reminder was sent to those identified as having 4 days of medication remaining. Finally, a text message or phone call reminder was sent 1 work day prior to all scheduled appointments.

Data collection involved obtaining from clinic's electronic medical record the number of attended versus no-showed psychiatric med checks from the identified child psychiatrist's 4 to 18 year old caseload, as well as the total number of med checks performed in the walk in clinic from that same population. For the purpose of this study, a no-show was defined as a psychiatric med check for which the parent, guardian, or identified client did not attend without advance same day call or notification. The clinic's documentation protocol for psychiatric med checks involved the identified clinician writing a med check progress note or indicating a no-show for every scheduled patient by the end of the work day, which was then automatically entered into the clinic's electronic medical record.

The no-show rate for the six month period of OCMOAS (2-1-2016 to 7-31-2016) was compared to the six months of traditional scheduling immediately prior to OCMOAS (8-1-2015 to 1-31-2016). In an attempt to minimize the possible impact of seasonal scheduling patterns on the results, the six months of OCMOAS was also compared to the coinciding six month period of traditional scheduling exactly 12 months prior (2-1-2015 to 7-31-2015). A onetailed $\mathrm{Z}$ test was used to test for statistical significance $($ alpha $=0.5)$.

\section{Results}

For the six month period immediately after the implementation of OCMOAS (2-1-2016 to 7-31-2016), 1440 med checks were scheduled of which 1156 were attended and 284 were no-showed, representing a 19.72\% no-show rate. For the six month period immediately prior (8-1-2015 to 1-31-2016) 1742 med checks were scheduled of which 1271 were attended and 471 were no-showed, representing a $27.04 \%$ no show rate. A $7.32 \%$ decrease in no-show rate was observed after the implementation of OCMOAS, the difference being statistically significant $(\mathrm{z}=4.8284, \mathrm{p}<0.0001)$.

For the six month period of 2-1-2015 to 7-31-2015, 1353 med checks were scheduled of which 1000 were attended and 353 were no-showed, representing a no-show rate of $26.10 \%$. This no-show rate is observed to be $6.38 \%$ higher than the six month period after OCMOAS was implemented, the difference being statistically significant $(\mathrm{z}=4.0084, \mathrm{p}<0.0001)$.

From 2-1-2016 to 7-31-2016 a total of 1244 med checks were seen, 88 of which came to the walk in clinic and 1156 were scheduled with the psychiatrist. Those seen in the walk-in clinic represented $7.07 \%$ of all med checks seen during this period.

\section{Discussion}

Preliminary interpretation of this data suggests that the employment of OCMOAS significantly decreased the noshow rate in this community mental health center's child and adolescent med check clinic. The findings are strengthened by the relatively large number of scheduled appointments reviewed and by the fact that statistical significance appeared to be unrelated to seasonal scheduling patterns.

The relatively low utilization rate of the walk in clinic is also an encouraging finding, as previous studies have associated open access scheduling with the potential loss of provider continuity ${ }^{10}$. Although the high rate of scheduling with the primary provider might be attributed to OCMOAS's ability to control the demand stream, an alternative explanation might suggest that this particular provider's caseload was already ideally suited in volume to accommodate a high rate of continuity.

Previous studies of open access scheduling have found improved patient experience and provider satisfaction ${ }^{11}$. Although patient and provider satisfaction with OCMOAS was not measured in this pilot study, an assessment of these factors would seem prudent before the OCMOAS model is applied on a larger scale. OCMOAS was met with resistance by some clients who preferred the option of being able to schedule routine appointments farther in advance, and some felt the inability to plan in advance for the med check to be a hardship. Furthermore, it was observed that client dissatisfaction tended to be unfairly displaced mostly onto the clerical staff responsible for implementing OCMOAS. On the other hand, many clients were appreciative of the greatly increased access to urgent or emergency med check appointments which had otherwise been difficult to obtain during the traditional scheduling phase.

\section{Limitations}

It is important to acknowledge the limitations of this study as it is based upon a sample of clients from only one outpatient clinic. Other study limitations include potential error in electronic medical record reporting, the unmeasured impact of requiring a face to face meeting before medications would be refilled, the utility of text messaging versus telephone calls for appointment reminders, the inability to correlate results with demographic data such as diagnosis, income, and insurance provider; and the undetermined number of emergency med checks being seen in the walk in clinic rather than by the primary provider. 


\section{Conclusion}

Due to the limitations of this study, the conclusions offered point toward the opportunity for further evaluation, rather than establishing definitive truths. The summation of limitations makes the pursuit of why the post-OCMOAS no-show rate remained at the high rate of $19.72 \%$ a matter of conjecture rather than certainty. The nature of no-shows in child and adolescent psychiatry is complicated, as it is generally a situation in which the parent either chooses or is unable to bring the child to the appointment for a combination of reasons; accordingly studies of missed appointments in child mental health treatment have found that most of the predictive variables were related to parent and family characteristics rather than to characteristics of the child ${ }^{12}$. Financial, transportation, social support, and motivational limitations are all too common among community mental health clients, and could be extenuating circumstances which force the parent to weigh the cost versus benefit of attending. Mental health impairments in parents such as depression and executive function difficulties may also be a factor in clients who persistently seem to forget their appointments ${ }^{13,14}$.

In summary, no-shows in child and adolescent psychiatry are a particularly concerning phenomenon that have ramifications beyond productivity and mental health outcomes. One might contend that children have a fundamental right to health care, and the failure to address the no-show phenomenon passively impedes that human right, as well as putting children at risk for negative health outcomes. Despite the limitations of this study, it can be argued that the findings, although preliminary, provide reason to be encouraged. The strongest attribute of OCMOAS ironically might be unrelated to technical innovation and more a function of greater outreach and communication between the clinic and client regarding scheduling issues. When taking into account the complexities that cause persistent no-show behaviors, OCMOAS deserves further research and development.

\section{References}

1. Thomas CR, Holzer CE. The Continuing Shortage of Child and Adolescent Psychiatrists. J Am Acad Child Adolesc Psychiatry. 2006;45(9):1-9.

2. Andrews R, Morgan JD, Addy DP, McNeish AS. Understanding non-attendance in outpatient paediatric clinics. Arch

3. Smoller JW, McLean RY, Otto MW and Pollock MH. How do clinicians respond to patients who miss appointments? J Clin Psychiatry. 1998;59(6):330338.

4. Molfenter T. Reducing appointment no-shows: going from theory to practice. Subst Use Misuse. 2013;48(9):743-749.
5. Steinman KJ, Shoben AB, Dembe AE, Kelleher KJ. How Long Do Adolescents Wait of Psychiatry Appointments? Community Ment Health J. 2015; 51(7):782-789.

6. Williams ME, Latta J, Conversano P. Eliminating the wait for mental health services. J Behav Health Serv Res. 2008;35(1):107-114.

7. Moldawsky R. Applying an Open-Access Model to a Psychiatric Practice. Perm J. 2007;11(1):33-36.

8. Murray M, Tantau C. Same-day appointments: exploding the access paradigm. Fam Pract Manag. 2000;7(8):45-50.

9. Lee S, Yih Y. Analysis of an Open Access Scheduling System in Outpatient Clinics: A Simulation Study. Simul. 2010;86(8):503-518.

10. Phan K, Brown SR. Decreased continuity in a residency clinic: a consequence of open access scheduling. Fam Med. 2009;41(1):46-50.

11. Boelke C, Boushon B, Isensee S. Achieving open access: the road to improved service \& satisfaction. Med Group Manage. 2000;47(5):64-66.

12. Peeters FP, Bayer H. 'No-Show' for initial screening at a community mental health centre: rate, reasons and further help-seeking. Soc Psychiatry Psychiatr Epidemiol. 1999;34(6):323-327.

13. Gordon M, Antshel KM, Lewandowski L, Seigers D. Predictors of Missed Appointments Over the Course of Child Mental Health Treatment. Psychiatr Serv. 2010;61(7):657-659.

14. Lesaca T. Executive functions in Parents with ADHD. Psychiatr Times. 2001;18(11):17-18. 